\title{
Pump-Pump-Probe Spectroscopy of a Molecular Triad Monitoring Detrimental Processes for Photoinduced Charge Accumulation
}

\author{
Martin Kuss-Petermann, ${ }^{a}$ and Oliver S. Wenger ${ }^{*, a}$ \\ a Department of Chemistry, University of Basel, St. Johanns-Ring 19, 4056 Basel, Switzerland, oliver.wenger@unibas.ch
}

\begin{abstract}
Controlling light-induced accumulation of electrons or holes is desirable in view of multi-electron redox chemistry, for example for the formation of solar fuels or for photoredox catalysis in general. Excitation with multiple photons is usually required for electron or hole accumulation, and consequently pump-pump-probe spectroscopy becomes a valuable spectroscopic tool. In this work, we excited a triarylamine-Ru(bpy) ${ }_{3}{ }^{2+}-$ anthraquinone triad (bpy $=2,2$ '-bipyridine) with two temporally delayed laser pulses of different color and monitored the resulting photoproducts. Absorption of the first photon by the $\mathrm{Ru}(\mathrm{bpy})_{3}{ }^{2+}$ photosensitizer generated a triarylamine radical cation and an anthraquinone radical anion by intramolecular electron transfer. Subsequent selective excitation of either one of these two radical ion species then induced rapid reverse electron transfer to yield the triad in its initial (ground) state. This shows in direct manner that after absorption of a first photon and formation of the primary photoproducts, the absorption of a second photon can lead to unproductive electron transfer events that counteract further charge accumulation. In principle, this problem is avoidable by careful excitation wavelength selection in combination with good molecular design.
\end{abstract}

Keywords: electron transfer • photochemistry • donor-acceptor systems $\bullet$ time-resolved spectroscopy $\bullet$ energy conversion

\section{Introduction}

In the vast majority of molecular electron transfer systems investigated to date, photoexcitation only leads to a simple electron-hole pair. For artificial photosynthesis, multi-electron redox chemistry and the accumulation of oxidative and reductive equivalents are highly relevant. ${ }^{[1]}$ Light-driven charge accumulation in molecular compounds is readily possible with sacrificial electron donors or acceptors, ${ }^{[2]}$ but ultimately the use of such energy-rich reagents is undesirable. ${ }^{[3]}$ Consequently, it seems important to explore the fundamentals of light-driven charge accumulation. Molecular compounds with covalently linked donors, photosensitizers, and acceptors are well suited for this purpose, but in absence of sacrificial reagents, light-driven charge accumulation becomes very challenging. ${ }^{[4-6]}$ To the best of our knowledge, there are currently only a handful of molecular systems in which charges were accumulated with light as an energy input without relying on sacrificial reagents. Two porphyrin-based triads were explored early and they both exhibited short-lived doubly reduced photoproducts $(\tau \leq 5 \mathrm{~ns}){ }^{[7]} \mathrm{In}$ a third study, the twofold reduction of a methylviologen unit relied on short-wavelength $(\lambda=266 \mathrm{~nm})$ photo-irradiation. ${ }^{\left[{ }^{8]}\right.} \mathrm{A}$ more recent system exhibited hole accumulation after excitation with visible light, but relied on $\mathrm{TiO}_{2}$ nanoparticles as electron acceptors. ${ }^{[9]}$ In molecular pentad I (Figure 1a) long-lived electron accumulation $(\tau=870 \mathrm{~ns}$ ) was achievable without sacrificial reagents with visible light as the sole energy input. ${ }^{[10]}$ Remarkably, that charge-accumulated state stored an energy of $\sim 3.5 \mathrm{eV}$, much more than commonly observed. ${ }^{[11]}$

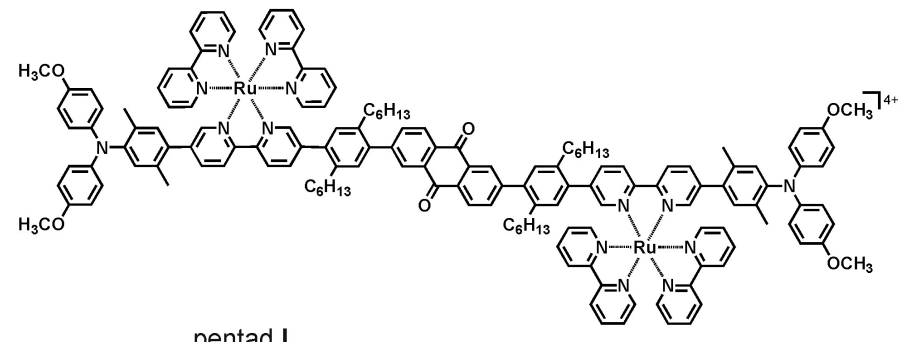

pentad I

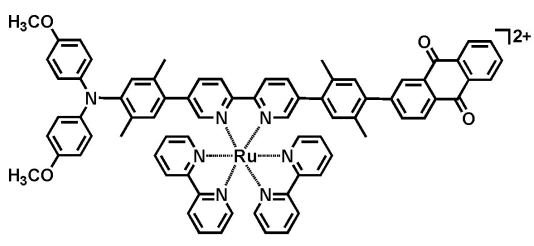

triad II

Figure 1. Molecular structures of pentad I (which is able to undergo photoinduced charge accumulation) ${ }^{[10]}$ and molecular triad II which was investigated in this work

Prior to reporting pentad $\mathbf{I}$, we attempted several times to achieve such light-driven charge accumulation in other molecular compounds, but failed to observe it unambiguously without sacrificial reagents. ${ }^{[12,13]}$ This is because many undesired reaction channels are open after absorption of a second photon by the same molecule, ${ }^{[4,5]}$ but we never probed any of these channels directly. In this work, we demonstrate the importance of one of these undesired processes by triggering it intentionally. Specifically, we use two temporally delayed laser pulses of different color for photoexcitation of molecular triad II (Figure 1b). This compound is unable to exhibit charge accumulation, ${ }^{[10]}$ but it is a simpler 
system than pentad $\mathbf{I}$, and it is well suited to study the process of interest in this study. After absorption of a first photon by the $\mathrm{Ru}(\mathrm{bpy})_{3}{ }^{2+}$ photosensitizer, an electron-hole pair is generated rapidly in the form of a triarylamine radical cation $\left(\mathrm{TAA}^{+}\right)$and an anthraquinone radical anion $\left(A Q^{-}\right)$. The wavelength of the second photon is chosen such that either $T A A^{+}$or $A Q^{-}$is excited selectively, and this induces rapid reverse electron transfer to result in the triad in its initial (ground) state. Thus, our pump-pump-probe experiment provides direct evidence for a process that can counteract light-induced charge accumulation. An earlier study investigated similar processes in the context of optical switching. ${ }^{[14]}$

\section{Results and Discussion}

Photoexcitation of triad II in $\mathrm{CH}_{3} \mathrm{CN}$ at $532 \mathrm{~nm}$ occurs selectively into the low-energy tail of the ${ }^{1} \mathrm{MLCT}$ absorption band of the Ru(bpy) ${ }_{3}^{2+}$ photosensitizer (Figure 2a). Previously we found that following intersystem crossing, the resulting ${ }^{3} \mathrm{MLCT}$ excited state is quenched reductively by TAA with a time constant of $9 \mathrm{ps}$, and subsequently electron transfer from the reduced sensitizer to AQ occurs with a time constant of 50 ps. ${ }^{[15]}$ The resulting charge-separated state comprised of $\mathrm{TAA}^{+}$and $\mathrm{AQ}^{-}$exhibits the transient absorption spectrum shown in Figure $2 \mathrm{~b}$.

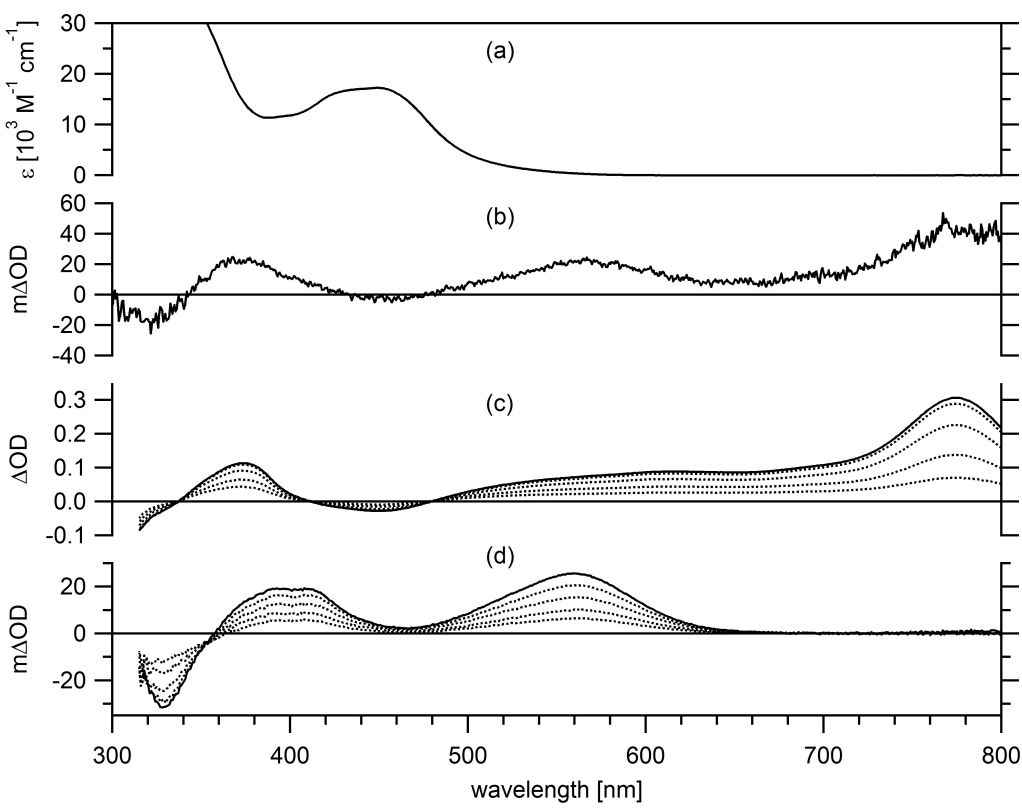

Figure 2. (a) Absorption spectrum of triad II in $\mathrm{CH}_{3} \mathrm{CN}$. (b) Transient absorption spectrum measured after excitation of $2 \cdot 10^{-5} \mathrm{M}$ triad II in de-aerated $\mathrm{CH}{ }_{3} \mathrm{CN}$ at 532 $\mathrm{nm}$ (the signal was integrated over a time interval of $200 \mathrm{~ns}$ immediately after excitation with laser pulses of $\sim 10 \mathrm{~ns}$ duration). (c) UV-Vis spectral changes observed when applying a potential of $+0.9 \mathrm{~V}$ vs.SCE to a $0.1 \mathrm{mM}$ solution of triad II in $\mathrm{CH}_{3} \mathrm{CN}$ with $0.1 \mathrm{M} \mathrm{TBAPF}_{6}$, monitoring the formation of TAA ${ }^{+}$. (d) UV-Vis spectral changes observed when applying a potential of $-1.0 \mathrm{~V}$ vs. SCE to the same solution, monitoring the formation of $A Q$.

Spectro-electrochemical data clearly confirm the formation of $\mathrm{TAA}^{+}$(bands at 370 and $775 \mathrm{~nm}$, Figure 2c) and AQ- (bands at 400 and $560 \mathrm{~nm}$, Figure 2d). This charge-separated state stores ca. $1.6 \mathrm{eV}$, and we previously estimated that nearly $100 \%$ of the triads that are excited to the ${ }^{3} \mathrm{MLCT}$ state end up in this singly charge-separated state. ${ }^{[15]}$ In de-aerated $\mathrm{CH}_{3} \mathrm{CN}$ at room temperature, the $\mathrm{TAA}^{+}$and $\mathrm{AQ}^{-}$spectral signatures both decay with a time constant of $1.6 \mu$ s (black traces in Figure 3), due to intramolecular thermal charge recombination. ${ }^{[15-18]}$

The black trace in Figure $3 a$ is an exemplary transient absorption decay detected at $770 \mathrm{~nm}\left(\mathrm{TAA}^{+}\right.$band) following excitation at $532 \mathrm{~nm}$ with laser pulses of $\sim 10 \mathrm{~ns}$ duration. The blue trace in Figure 3a results from a pump-pump-probe experiment in which a first series of laser pulses excited the triad at $532 \mathrm{~nm}$, followed by a second series of laser pulses which excited the same sample at $450 \mathrm{~nm}$ with a time delay of $400 \mathrm{~ns}$. At $450 \mathrm{~nm}$, the photosensitizer in its ground state absorbs strongly (Figure 2a), while the $\mathrm{TA}^{+}$and $\mathrm{AQ}^{-}$radical species exhibit very little change in absorption with respect to the ground state (Figure 2b). The pulse energies used for the two lasers were $18 \mathrm{~mJ}$ at $532 \mathrm{~nm}$ and 12 $\mathrm{mJ}$ at $450 \mathrm{~nm}$, yet the second series of pulses produces substantially increased transient absorption at $770 \mathrm{~nm}$ (blue trace in Figure $3 a$ ). The transient absorption spectrum recorded after the second series of pulses exhibits no spectral changes with regard to the transient absorption spectrum measured after the first series of pulses (Figure S1). This indicates that the second series of pulses does not lead to new photoproducts but simply produces more of the same. A substantial amount of additional $\mathrm{TAA}^{+} / \mathrm{AQ}^{-}$pairs forms because the extinction coefficient of the MLCT excited state is significantly higher at $450 \mathrm{~nm}$ than at $532 \mathrm{~nm}$ (Figure 2a). Transients at detection wavelengths of 375 $\mathrm{nm}$ (monitoring TAA ${ }^{+}$and $\mathrm{AQ}^{-}$, Figure S2a) and $560 \mathrm{~nm}$ (monitoring $\mathrm{AQ}^{-}$, Figure $\mathrm{S} 2 \mathrm{~d}$ ) are analogous to that in Figure 3a. 

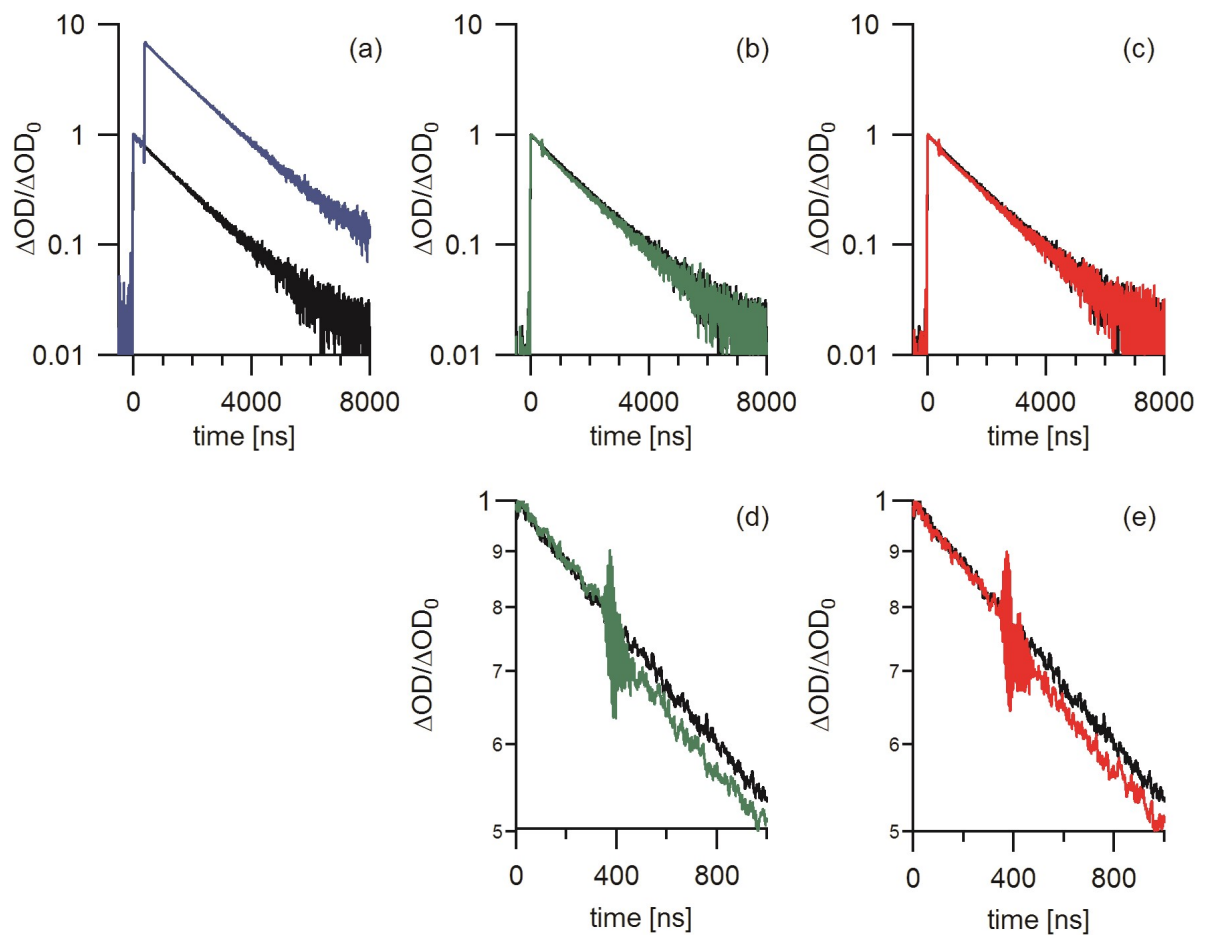

Figure 3. Temporal evolution of transient absorption signals recorded from a $2 \cdot 10^{-5} \mathrm{M}$ solution of triad II in de-aerated $\mathrm{CH}_{3} \mathrm{CN}$ at room temperature. Primary excitation always occurred at $532 \mathrm{~nm}$ with laser pulses of $\sim 10 \mathrm{~ns}$ duration, detection was always at $770 \mathrm{~nm}$ (for other detection wavelengths see the Supporting Information). Wavelengths used for secondary excitation were $450 \mathrm{~nm}$ (blue), $580 \mathrm{~nm}$ (green), and $810 \mathrm{~nm}$ (red). The time delay between the two series of excitation pulses was always $400 \mathrm{~ns}$. The black traces result from ordinary pump-probe experiments using 532-nm excitation without subsequent secondary excitation. Panels (d) and (e) contain the data from panels (b) and (c) on enlarged scales.

The black trace in Figure $3 \mathrm{~b}$ is again a transient decay measured at $770 \mathrm{~nm}$ after excitation with $532 \mathrm{~nm}$ pulses only, whereas the superimposed green decay results from a pump-pump-probe experiment in which the initial $532 \mathrm{~nm}$ series of pulses (18 mJ) was followed by a $580 \mathrm{~nm}$ pulse series $(10 \mathrm{~mJ})$. At $580 \mathrm{~nm}$, there is essentially no absorption of the triad in its ground state (Figure 2a) but $A Q^{-}$exhibits an absorption maximum near this wavelength in neat $\mathrm{CH}_{3} \mathrm{CN}$ (Figure $2 \mathrm{~b}, \mathrm{~d}$ ). Thus, only the fraction of triads which was promoted to the TAA+ / $A Q^{-}$charge-separated state with the first series of pulses can be excited with the second pulse series, and only a subset of this fraction will indeed absorb a second photon. Consequently, the change in the green transient with respect to the black one is small and becomes only visible in Figure $3 d$, in which the data from Figure $3 b$ are shown on enlarged scales. In Figure 3d the two decays are essentially identical in the first $400 \mathrm{~ns}$, and then the green trace exhibits some noise (presumably of electronic origin) marking the pulsing of the second laser. Beyond $450 \mathrm{~ns}$ the black and the green transients decay in parallel, but the green transient now has about $5 \%$ diminished intensity relative to the black one. This indicates that a portion of the TAA ${ }^{+}$radical cations which were formed with the first pulse series have disappeared after the second series of pulses. Very similar transients are detectable when monitoring $\mathrm{AQ}^{-}$at $560 \mathrm{~nm}$ or the combination of TAA ${ }^{+}$and $\mathrm{AQ}^{-}$at $375 \mathrm{~nm}\left(\mathrm{Figure}^{-}\right.$ $\mathrm{S} 3 \mathrm{~b}, \mathrm{e})$. When recording the full transient absorption spectrum by time-integrating over 200 ns following the second series of laser pulses, no spectral changes with respect to the spectrum obtained after the first series of pulses are observable (Figure S1), again indicating that no new photoproducts are formed with the second pulse series. We conclude that excitation of $A^{-}$at $580 \mathrm{~nm}$ causes rapid (<50 ns) charge recombination between $\mathrm{AQ}^{-}$and $\mathrm{TAA}^{+}$. We estimate that ca. $29 \%$ of all triads in the initially pumped sample volume are promoted to the $\mathrm{TAA}^{+} /$ $\mathrm{AQ}^{-}$charge-separated state (see Supporting Information for details) under the conditions used, and out of these $\sim 29 \%$ only $\sim 5 \%$ then undergo photoinduced charge recombination in consequence of direct $A Q^{-}$excitation.

Analogous observations are made in a pump-pump-probe experiment in which initial excitation at $532 \mathrm{~nm}(18 \mathrm{~mJ})$ is followed by subsequent selective excitation of $\mathrm{TAA}^{+}$at $810 \mathrm{~nm}$ (Figure $\left.3 \mathrm{c}, \mathrm{e}\right)$. The pulses at $810 \mathrm{~nm}(8.5 \mathrm{~mJ})$ occurring with a delay of $400 \mathrm{~ns}$ cause a rapid (< $\left.50 \mathrm{~ns}\right)$ decrease of approximately $5 \%$ of the transient absorption signals at $770 \mathrm{~nm}$ (Figure 3e), $375 \mathrm{~nm}$ (Figure S3c) and at $560 \mathrm{~nm}$ (Figure S3f). In the time-integrated transient absorption spectrum, no significant spectral differences appear with respect to the ordinary pump-probe experiment (Figure S1). We conclude that excitation of $\mathrm{TAA}^{+}$causes charge recombination between $\mathrm{AQ}^{-}$and $\mathrm{TAA}^{+}$, in analogy to what is observed when exciting $A Q$ :

The mechanism for photoinduced charge recombination cannot be inferred from the available data, and the excited states of TAA ${ }^{+}$and $A Q^{-}$are very short-lived. ${ }^{[19]}$ However, photoexcited $\mathrm{TAA}^{+}$is expected to be a very strong acceptor while photoexcited $A Q^{-}$should be a very strong donor. ${ }^{[20]}$ It is conceivable that these species are in fact sufficiently potent redox reagents for (temporary) oxidation or reduction of the central $\mathrm{Ru}(\mathrm{bpy})_{3}{ }^{2+}$ photosensitizer unit, and this would provide an efficient charge recombination pathway resembling a hopping mechanism. ${ }^{[21]}$ 


\section{Conclusions}

The absorption of a first photon and the formation of primary photoproducts in the course of a light-induced charge accumulation process leads to changes in the absorption spectrum, and when a second photon is then absorbed by the photoproducts, this may lead to undesired reactions. This is demonstrated here in direct manner by intentionally exciting into the primary photoproducts of triad II using two-color pumppump-probe experiments. Triad II cannot undergo photodriven charge accumulation, but it is a well-suited model compound for the demonstration of the principle of photoinduced charge recombination.

The effect of the second pulse looks small at first glance (Figure 3), but two-photon experiments normally exhibit quadratic power dependences. ${ }^{[10,22]}$ We estimate that $\sim 29 \%$ of the triads in the pumped sample volume are promoted to the $\mathrm{TAA}^{+} / \mathrm{AQ}^{-}$state with the first series of pulses under the conditions used here, but only $\sim 5 \%$ of this subset of molecules is then undergoing photoinduced charge recombination while $\sim 95 \%$ exhibit ordinary thermal charge recombination. In compounds that are set up for photoinduced charge accumulation, the percentage of molecules that can undergo absorption of a second photon is typically on the same order of magnitude hence the effect observed here is significant. ${ }^{[7,9,10]}$

In compounds designed specifically for charge accumulation it is normally more straightforward to use a single laser pulse of sufficient power rather than two temporally delayed excitation pulses of different color. For solar energy conversion, for example when charge accumulation is intended to occur on a catalytically active reaction center, then it is most likely desirable to perform continuous excitation in a specific wavelength range. For such applications, it becomes necessary to optimize donors, sensitizers, acceptors, and excitation wavelengths such that energy-wasting processes resulting from excitation of the primary photoproducts are minimized. Proper choice of the photosensitizer is particularly crucial in this regard, and it is desirable to know the spectroscopic signatures of the primary photoproducts.

In charge accumulation schemes, yet other unproductive and energy-wasting processes can be triggered upon absorption of a second photon. ${ }^{[5,13]}$ In triad II, specific key processes in this regard are reductive quenching of the photoexcited sensitizer unit by $\mathrm{AQ}^{-}$and oxidative quenching by $\mathrm{TAA}^{+}$. There is no direct evidence for these two processes in our study (Figure 3a) because excitation with the second photon at $450 \mathrm{~nm}$ into the MLCT absorption of the sensitizer results in a large amount of additional $\mathrm{TAA}^{+} / \mathrm{AQ}^{-}$photoproducts, which overcompensates eventual losses by the abovementioned processes. In prior studies such processes seemed indeed detrimental, ${ }^{[13,23]}$ and we cannot exclude that they also play an important role in triad II. Thus, while our study is able to provide direct evidence for photoinduced charge recombination, other detrimental processes involved in charge accumulation schemes are still to be probed in further detail.

\section{Experimental Section}

The synthesis of triad II and its electrochemical properties were reported in a prior study. ${ }^{[16]}$ Spectro-electrochemistry occurred as described previously. ${ }^{[18]}$ Basic transient absorption studies of triad II (involving single excitation) were made in prior work. ${ }^{[15-17, ~ 18, ~ 24]}$ The two-color pumppump probe experiments reported herein were performed on an LP920-KS instrument from Edinburgh Instruments using the frequencydoubled output of a Quantel Brilliant b laser for the first excitation, and a Quantel Brilliant laser equipped with an OPO from Opotek for the second excitation. Synchronization of the two lasers occurred with a 9520 digital delay pulse generator from Quantum Composers.

\section{Supplementary Material}

Supporting information for this article is available on the WWW under http://dx.doi.org/10.1002/MS-number.

\section{Acknowledgements}

This work was supported by the Swiss National Science Foundation through grant number 200021-146231/1 and the NCCR Molecular Systems Engineering.

\section{Author Contribution Statement}

M. K.-P. and O. S. W. conceived this work and designed experiments. M. K.-P. performed experiments. M. K.-P. and O. S. W. jointly analyzed and interpreted data and wrote the paper.

\section{References}

[1] D. Gust, T. A. Moore, A. L. Moore, 'Solar Fuels via Artificial Photosynthesis', Acc. Chem. Res. 2009, 42, 1890-1898; T. J. Meyer, 'Chemical Approaches to Artificial Photosynthesis', Acc. Chem. Res. 1989, 22, 163-170.

[2] R. Konduri, H. W. Ye, F. M. MacDonnell, S. Serroni, S. Campagna, K. Rajeshwar, 'Ruthenium Photocatalysts Capable of Reversibly Storing up to Four Electrons in a Single Acceptor Ligand: A Step Closer to Artificial Photosynthesis', Angew. Chem. Int. Ed. 2002, 41, 3185-3187; K. L. Wouters, N. R. de Tacconi, R. Konduri, R. O. Lezna, F. M. MacDonnell, 'Driving Multi-Electron Reactions with Photons: Dinuclear Ruthenium Complexes Capable of Stepwise and Concerted Multi-Electron Reduction', Photosynth. Res. 2006, 87, 41-55; R. Konduri, N. R. de Tacconi, K. Rajeshwar, F. M. MacDonnell, 'Multielectron 
Photoreduction of a Bridged Ruthenium Dimer, (phen $)_{2} R u(\operatorname{tatpp}) \mathrm{Ru}(\mathrm{phen})_{2}\left(\mathrm{PF}_{6}\right)_{4}$ : Aqueous Reactivity and Chemical and Spectroelectrochemical Identification of the Photoproducts', J. Am. Chem. Soc. 2004, 126, 11621-11629; G. F. Manbeck, K. J. Brewer, 'Photoinitiated Electron Collection in Polyazine Chromophores Coupled to Water Reduction Catalysts for Solar $\mathrm{H}_{2}$ Production', Coord. Chem. Rev. 2013, 257, 1660-1675; M. Elvington, K. J. Brewer, 'Photoinitiated Electron Collection at a Metal in a Rhodium-Centered Mixed-Metal Supramolecular Complex', Inorg. Chem. 2006, 45, 5242-5244; S. M. Molnar, G. Nallas, J. S. Bridgewater, K. J. Brewer, 'Photoinitiated Electron Collection in a Mixed-Metal Trimetallic Complex of the Form $\left\{\left[(\mathrm{bpy})_{2} \mathrm{Ru}(\mathrm{dpb})\right]_{2} \mathrm{IrCl}_{2}\right\}\left(\mathrm{PF}_{6}\right)_{5}\left(\mathrm{bpy}=2,2^{\prime}-\mathrm{Bipyridine}\right.$ and dpb = 2,3-bis(2-Pyridyl)benzoquinoxaline)', J. Am. Chem. Soc. 1994, 116, 5206-5210; C. Chiorboli, S. Fracasso, F. Scandola, S. Campagna, S. Serroni, R. Konduri, F. M. MacDonnell, 'Primary Charge Separation in Photoinduced Multielectron Storage Systems. A Dinuclear Ruthenium(II) Species Featuring a Charge-Separated State with a Lifetime of 1.3 $\mu$ ', Chem. Commun. 2003, 1658-1659.

[3] Y. Pellegrin, F. Odobel, 'Sacrificial Electron Donor Reagents for Solar Fuel Production', C. R. Chimie 2016, doi: 10.1016/j.crci.2015.1011.1026.

[4] Y. Pellegrin, F. Odobel, 'Molecular Devices Featuring Sequential Photoinduced Charge Separations for the Storage of Multiple Redox Equivalents', Coord. Chem. Rev. 2011, 255, 2578-2593.

[5] L. Hammarström, 'Accumulative Charge Separation for Solar Fuels Production: Coupling Light-Induced Single Electron Transfer to Multielectron Catalysis', Acc. Chem. Res. 2015, 48, 840-850.

[6] A. G. Bonn, O. S. Wenger, 'Photoinduced Charge Accumulation in Molecular Systems', Chimia 2015, 69, 17-21.

[7] M. P. O'Neil, M. P. Niemczyk, W. A. Svec, D. Gosztola, G. L. Gaines, M. R. Wasielewski, 'Picosecond Optical Switching Based on Biphotonic Excitation of an Electron Donor-Acceptor-Donor Molecule', Science 1992, 257, 63-65; H. Imahori, M. Hasegawa, S. Taniguchi, M. Aoki, T. Okada, Y. Sakata, 'Synthesis and Photophysical Properties of Porphyrin-Tetracyanoanthraquinodimethane-Porphyrin Triad: Photon-Dependent Molecular Switching', Chem. Lett. 1998, 721-722.

[8] T. H. Ghaddar, J. F. Wishart, D. W. Thompson, J. K. Whitesell, M. A. Fox, 'A Dendrimer-Based Electron Antenna: Paired Electron-Transfer Reactions in Dendrimers with a 4,4 '-Bipyridine Core and Naphthalene Peripheral Groups', J. Am. Chem. Soc. 2002, 124, 8285-8289.

[9] S. Karlsson, J. Boixel, Y. Pellegrin, E. Blart, H. C. Becker, F. Odobel, L. Hammarström, 'Accumulative Electron Transfer: Multiple Charge Separation in Artificial Photosynthesis', Faraday Discuss. 2012, 155, 233-252; S. Karlsson, J. Boixel, Y. Pellegrin, E. Blart, H. C. Becker, F. Odobel, L. Hammarström, 'Accumulative Charge Separation Inspired by Photosynthesis', J. Am. Chem. Soc. 2010, 132, 17977-17979.

[10] M. Orazietti, M. Kuss-Petermann, P. Hamm, O. S. Wenger, 'Light-Driven Electron Accumulation in a Molecular Pentad', Angew. Chem. Int. Ed. 2016, 55, 9407-9410.

[11] L. Favereau, A. Makhal, Y. Pellegrin, E. Blart, J. Petersson, E. Goransson, L. Hammarström, F. Odobel, 'A Molecular Tetrad That Generates a High-Energy Charge-Separated State by Mimicking the Photosynthetic Z-Scheme', J. Am. Chem. Soc. 2016, 138, 3752-3760; G. H. Lim, C. O. Obondi, F. D'Souza, 'A High-Energy Charge-Separated State of $1.70 \mathrm{eV}$ from a High-Potential Donor-Acceptor Dyad: A Catalyst for Energy-Demanding Photochemical Reactions', Angew. Chem. Int. Ed. 2016, doi: 10.1002/anie.201606112.

[12] A. G. Bonn, M. Neuburger, O. S. Wenger, 'Photoinduced Electron Transfer in Rhenium(I)-Oligotriarylamine Molecules', Inorg. Chem. 2014, 53, 1107511085; A. G. Bonn, O. S. Wenger, 'Photoinduced Charge Accumulation by Metal Ion-Coupled Electron Transfer', Phys. Chem. Chem. Phys. 2015, 17, 24001-24010.

[13] A. G. Bonn, O. Yushchenko, E. Vauthey, O. S. Wenger, 'Photoinduced Electron Transfer in an Anthraquinone- Ru(bpy) $3^{2+}{ }^{-O}$ ligotriarylamine-Ru(bpy $3_{3}{ }^{2+}$ Anthraquinone Pentad', Inorg. Chem. 2016, 55, 2894-2899.

[14] M. P. Debreczeny, W. A. Svec, E. M. Marsh, M. R. Wasielewski, 'Femtosecond Optical Control of Charge Shift within Electron Donor-Acceptor Arrays: An Approach to Molecular Switches', J. Am. Chem. Soc. 1996, 118, 8174-8175.

[15] J. Hankache, M. Niemi, H. Lemmetyinen, O. S. Wenger, 'Photoinduced Electron Transfer in Linear Triarylamine-Photosensitizer-Anthraquinone Triads with Ruthenium(II), Osmium(II), and Iridium(III)', Inorg. Chem. 2012, 51, 6333-6344.

[16] J. Hankache, O. S. Wenger, 'Microsecond Charge Recombination in a Linear Triarylamine-Ru(bpy) $3^{2+}-$ Anthraquinone Triad', Chem. Commun. 2011, 47, 10145-10147.

[17] J. Hankache, O. S. Wenger, 'Large Increase of the Lifetime of a Charge-Separated State in a Molecular Triad Induced by Hydrogen-Bonding Solvent', Chem. Eur. J. 2012, 18, 6443-6447.

[18] M. Kuss-Petermann, O. S. Wenger, 'Electron Transfer Rate Maxima at Large Donor-Acceptor Distances', J. Am. Chem. Soc. 2016, 138, 1349-1358; M. Kuss-Petermann, O. S. Wenger, 'Increasing Electron-Transfer Rates with Increasing Donor-Acceptor Distance', Angew. Chem. Int. Ed. 2016, 55, 815-819.

[19] A. R. Cook, L. A. Curtiss, J. R. Miller, 'Fluorescence of the 1,4-Benzoquinone Radical Anion', J. Am. Chem. Soc. 1997, 119, 5729-5734; M. Zamadar, A. R. Cook, A. Lewandowska-Andralojc, R. Holroyd, Y. Jiang, J. Bikalis, J. R. Miller, 'Electron Transfer by Excited Benzoquinone Anions: Slow Rates for TwoElectron Transitions', J. Phys. Chem. A 2013, 117, 8360-8367; M. Fujita, A. Ishida, T. Majima, S. Takamuku, 'Lifetimes of Radical Anions of Dicyanoanthracene, Phenazine, and Anthraquinone in the Excited State from the Selective Electron-Transfer Quenching', J. Phys. Chem. 1996, 100, 53825387; P. Brodard, A. Sarbach, J. C. Gumy, T. Bally, E. Vauthey, 'Excited-State Dynamics of Organic Radical lons in Liquids and in Low-Temperature Matrices', J. Phys. Chem. A 2001, 105, 6594-6601.

[20] M. Sakamoto, X. C. Cai, S. S. Kim, M. Fujitsuka, T. Majima, 'Intermolecular Electron Transfer from Excited Benzophenone Ketyl Radical', J. Phys. Chem. A 2007, 111, 223-229; M. Fujitsuka, S. S. Kim, C. Lu, S. Tojo, T. Majima, 'Intermolecular and Intramolecular Electron Transfer Processes from Excited Naphthalene Diimide Radical Anions', J. Phys. Chem. B 2015, 119, 7275-7282; D. T. Breslin, M. A. Fox, 'Excited-State Behavior of Thermally Stable Radical lons', J. Phys. Chem. 1994, 98, 408-411.

[21] M. Cordes, B. Giese, 'Electron Transfer in Peptides and Proteins', Chem. Soc. Rev. 2009, 38, 892-901.

[22] M. Pollnau, D. R. Gamelin, S. R. Lüthi, H. U. Güdel, M. P. Hehlen, 'Power Dependence of Upconversion Luminescence in Lanthanide and Transition-MetalIon Systems', Phys. Rev. B 2000, 61, 3337-3346; A. Haefele, J. Blumhoff, R. S. Khnayzer, F. N. Castellano, 'Getting to the (Square) Root of the Problem: How to Make Noncoherent Pumped Upconversion Linear', J. Phys. Chem. Lett. 2012, 3, 299-303.

[23] A. Magnuson, M. Anderlund, O. Johansson, P. Lindblad, R. Lomoth, T. Polivka, S. Ott, K. Stensjö, S. Styring, V. Sundström, L. Hammarström, 'Biomimetic and Microbial Approaches to Solar Fuel Generation', Acc. Chem. Res. 2009, 42, 1899-1909; M. Borgström, N. Shaikh, O. Johansson, M. F. Anderlund, S. 


\section{HELVETICA}

Styring, B. Ákermark, A. Magnuson, L. Hammarström, 'Light Induced Manganese Oxidation and Long-Lived Charge Separation in a $\mathrm{Mn}_{2}{ }^{\mathrm{Il}, \mathrm{I}}-\mathrm{Ru}^{\mathrm{I}}(\mathrm{bpy})_{3}-$ Acceptor Triad', J. Am. Chem. Soc. 2005, 127, 17504-17515.

[24] J. Hankache, M. Niemi, H. Lemmetyinen, O. S. Wenger, 'Hydrogen-Bonding Effects on the Formation and Lifetimes of Charge-Separated States in Molecular Triads', J. Phys. Chem. A 2012, 116, 8159-8168. 
Entry for the Table of Contents

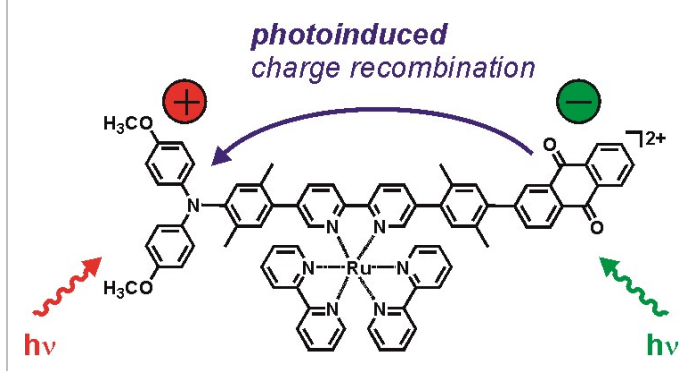

